\title{
Weak faults at megathrust plate boundary respond to tidal stress
}

\author{
Takashi Tonegawa ${ }^{1 *}{ }^{*}$, Toshinori Kimura ${ }^{1}$, Kazuya Shiraishi ${ }^{1}$, Suguru Yabe ${ }^{2}$, Yoshio Fukao ${ }^{1}$, Eiichiro Araki ${ }^{1}$, \\ Masataka Kinoshita ${ }^{3}$, Yoshinori Sanada' ${ }^{1}$ Seiichi Miura', Yasuyuki Nakamura ${ }^{1}$ and Shuichi Kodaira ${ }^{1}$
}

\begin{abstract}
Lateral spatial variations of weak portions at the plate boundary in subduction zones have been estimated primarily by the distribution of slow earthquakes mainly occurring around seismogenic zones. However, the detailed depth profile of weak faults remains elusive. Here, we deployed six ocean bottom seismometers in the Nankai subduction zone, Japan, to observe reflections originated from drilling vessel Chikyu ship noise (hydroacoustic $P$ wave) that was persistently radiated from a fixed position at the sea surface, and retrieved $P$-to-s $\left(P_{S}\right)$ reflections from multiple dipping faults near the plate boundary. The $P$ s amplitudes were stacked and compared according to the degrees of tidal stresses, and high amplitudes were observed at high tide (compression). A migration technique shows that the locations where velocity contrasts fluctuate were estimated at both the megasplay fault and another fault between the megasplay fault and the top of the oceanic crust. This indicates that the physical properties of these faults are altered by tidal stress. The physical-property changes are attributed to fluid connections and isolations within the faults due to tidal stress fluctuations, inducing the variation of seismic anisotropy. Such a variation was confirmed by a threedimensional numerical simulation for wave propagation with anisotropic medium. Our observation suggests that multiple weak faults are present around the plate boundary, and the obtained changes of fault physical properties may have implications for in-depth understanding of tidal triggering of earthquakes.
\end{abstract}

Keywords: Ambient noise, Seafloor observation, Ps reflection, Ship noise, Tidal response, Megasplay fault

\section{Introduction}

Tidal stresses result from the deformation of the Earth and ocean loading due to the gravitational pull of the Sun and Moon, and these can trigger ordinary (Cochran et al. 2004; Tanaka et al. 2006; Ide et al. 2016) and slow earthquakes (Rubinstein et al. 2008; Nakata et al. 2008; Lambert et al. 2009; Ide 2010) by stress fluctuations on plate boundaries in subduction zones. The tidal sensitivity of slow earthquakes is affected by the fluids within faults (Hawthorne and Rubin 2010; Houston 2015; Yabe et al. 2015; Nakamura and Kakazu 2017). Under wetter conditions, the physical properties of the plate boundary and

\footnotetext{
*Correspondence: tonegawa@jamstec.go.jp

1 Japan Agency for Marine-Earth Science and Technology (JAMSTEC),

2-15, Natsushima-cho, Yokosuka 237-0061, Japan

Full list of author information is available at the end of the article
}

thereby its frictional properties are presumably affected by external periodic forces. However, there has been little observation on the tidal response of plate boundary properties because it requires continuous natural or artificial seismic sources as well as persistent retrieval of seismic waves sampling the boundary. The discovery of such phenomena could allow for the identification of mechanically weak faults that are closely linked to rupture propagations of megathrust earthquakes and tsunami generation, and this could be the key to understanding tidal triggering of earthquakes.

A scientific drilling project was carried out between October 2018 and March 2019 by the International Ocean Discovery Program (IODP) with the drilling vessel D/V Chikyu (hereafter Chikyu) (IODP expedition 358) (Fig. 1) at the C0002 site located off the Kii Peninsula in the Nankai subduction zone, Japan, beneath which 

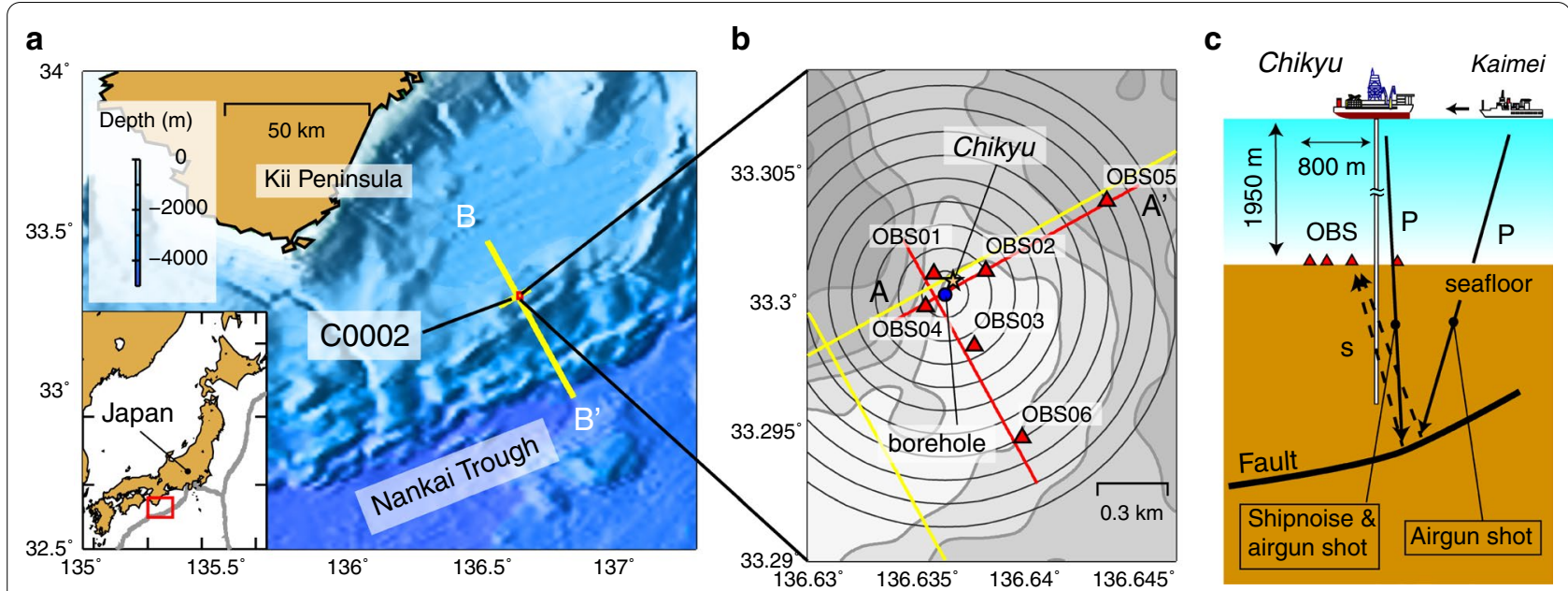

Fig. 1 Geometry of the Chikyu, drilling site, and OBSs. a Location of the drilling site (C0002) and reflection profiles (Shiraishi et al. 2019) (yellow lines). $\mathbf{b}$ The red triangles show the locations of the six OBSs. The red lines show the locations of the migration images shown in Figs. 7 and 8 . The yellow lines correspond to the reflection profiles used in Figs. 7 and 8 (Shiraishi et al. 2019). c Vertical cross-section showing the Chikyu, the Kaimei, the OBSs and the fault at depth. Solid and dashed lines indicate $P$ and $S$ waves, respectively

recurring slow slip events have occurred around the plate boundary (Araki et al. 2017). Because the Chikyu has the potential to excite incessant seismic sources during the project, we installed six ocean bottom seismometers (OBSs) at distances ranging between 100 and $800 \mathrm{~m}$ from the drilling site in the period from November 2018 to May 2019 to retrieve seismic reflections beneath the seafloor originated from the Chikyu.

Seismic interferometry is capable of detecting waves propagating between two receivers by cross-correlating the ambient noise recorded at both the receivers (Shapiro 2005; Brenguier et al. 2007). Applying this technique to continuous seismic records observed at a near-vertical array of OBS04 and a borehole sensor (Kopf et al. 2011, 2016) (Fig. 1b), we found that the downgoing $P$ waves generated by the Chikyu ship noise were dominant during the non-drilling period, while the upgoing $S$ waves from the drill-bit torque at depth could be observed in the drilling period (See details in "Materials and methods" section): Chikyu produces hydroacoustic $P$ waves in the sea water by, e.g., internal instrumental and external thruster noises, from the fixed drilling position at the sea surface, and they are converted to downgoing $P$ waves at the seafloor. Therefore, we examined the Chikyu shipnoise records acquired by the 6 OBSs during the nondrilling periods of the project, to persistently retrieve the seismic waves reflected from the downgoing $P$ waves at the deep structure around the plate boundary.

In the "Materials and methods" section, we confirm the wavefield near the seafloor during the non-drilling period with seafloor and borehole sensors, correct the amplitudes of the six OBSs with site amplifications, and extract reflections through a deconvolution technique from both airgun shot experiments conducted by the two vessels and the Chikyu ship noise. In the "Results" section, we compare the amplitude variations of reflections in the deconvolved waveforms using the calculated tidal stresses, and image the locations, where the effects of tidal stress can be observed, through a migration technique. We finally discuss a possible model that explains reflection amplitudes induced by tidal stresses.

\section{Materials and methods}

\section{Ambient noise wavefield during the drilling period}

Six ocean-bottom seismometers (OBSs) were installed at a range of distances between 100 and $800 \mathrm{~m}$ from $\mathrm{C} 0002$. Four OBSs were placed at 100, 100, 200 and $250 \mathrm{~m}$ by the remotely operated vehicle (ROV) installed on Chikyu and the two remaining OBSs were dropped in free-fall from $\mathrm{R} / \mathrm{V}$ Yokosuka at 700 and $800 \mathrm{~m}$ from the drilling site; their location errors are $\sim 2 \mathrm{~m}$ and $10-20 \mathrm{~m}$, respectively.

To investigate the ambient noise wavefield during the drilling project, we calculated the cross-correlation function (CCF) of continuous records observed by $\mathrm{OBS} 04$ and a borehole sensor that is deployed at a depth of $900 \mathrm{~m}$ beneath the seafloor (Figs. $1 \mathrm{~b}$ and 2a) (Kopf et al. 2011, 2016). The OBS contains a $4.5 \mathrm{~Hz}$ short-period sensor with three components. The length of the time window was $80 \mathrm{~s}$. Spectral whitening was performed during the calculation of the CCF (Brenguier et al. 2007; Bensen et al. 2007). Because the rotation speed information of the drill-bit (RPM: rotation per minute) in the drilling vessel Chikyu was available (Fig. 2b), the CCFs averaged over a 1-hour period using ambient noise records of the 


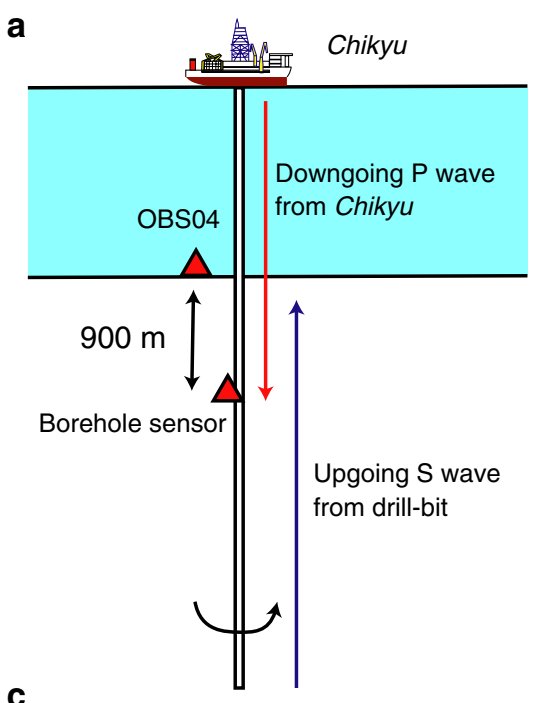

C

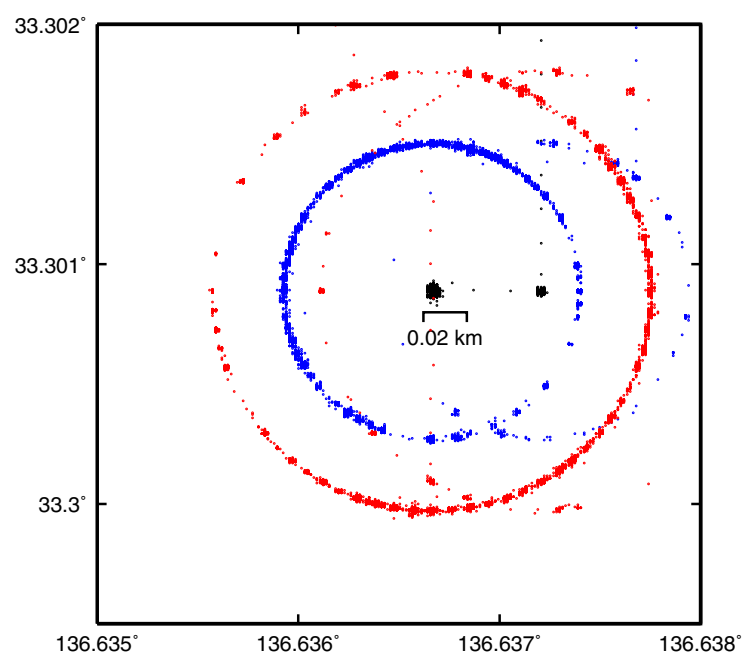

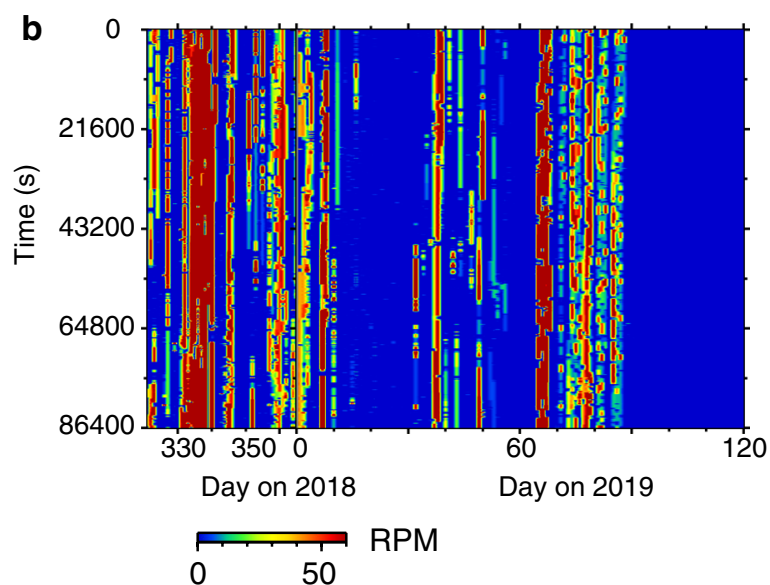

d

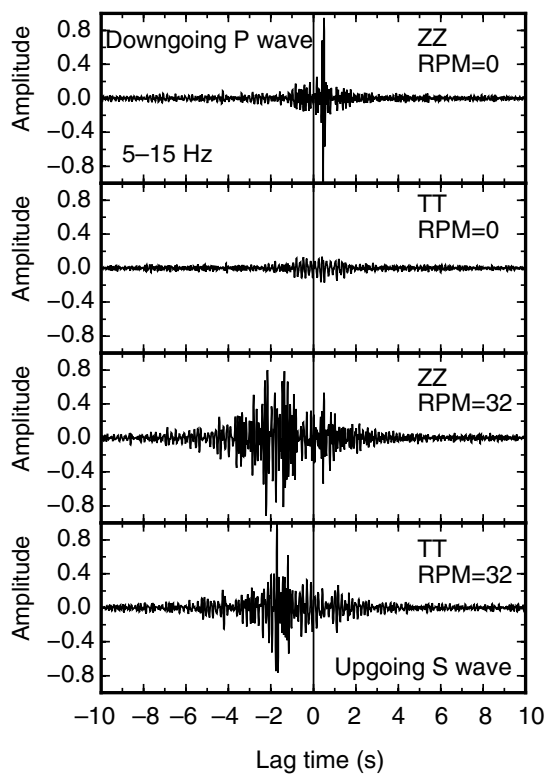

Fig. 2 Ambient noise field during the drilling project. a Geometry of DN Chikyu, OBSO4, and borehole sensor. b Rotation per minute (RPM) of drill-bit during the drilling project. The vertical axis is time over a 1-day period. c The blue, black, and red dots represent the locations of the front, center, and back parts of the Chikyu during the Julian days 1-58 in 2019. d Waveforms of (from top to bottom) ZZ-CCF (cross correlation function) for $\mathrm{RPM}=0, \mathrm{TT}-\mathrm{CCF}$ for $\mathrm{RPM}=0, \mathrm{ZZ}-\mathrm{CCF}$ for $\mathrm{RPM}=32$, and TT-CCF for RPM $=32$. The reference site of the CCFs is OBSO4, and indicates that peaks at positive/negative lag time are downgoing/upgoing waves

vertical component $(\mathrm{ZZ})$ between the two sensors were calculated under two conditions: 1-hour averaged RPMs of 0 (non-drilling period: 10:00-11:00 (UT), November 20, 2018) and 32 (drilling period: 13:00-14:00 (UT), November 20, 2018). When RPM $=32$, the drill-bit was located at a depth of $\sim 5000 \mathrm{~m}$ from the sea surface (Additional file 1: Fig. S1). This processing was also performed using the transverse component (TT), in which the transverse direction at the two sensors is perpendicular to the directions from the Chikyu to the two sensors.
Figure $2 \mathrm{~d}$ shows four $\mathrm{CCFs}(\mathrm{ZZ}$ and $\mathrm{RPM}=0, \mathrm{ZZ}$ and $\mathrm{RPM}=32$, TT and $\mathrm{RPM}=0$, and TT and $\mathrm{RPM}=32$ ). Because the reference site is OBS04 (seafloor) for the CCF calculation, peaks with large amplitudes at the positive/negative lag time in the CCFs represent the downgoing/upgoing wave. The ZZ-CCF for RPM $=0$ shows the downgoing $P$ wave at a lag time of $0.5 \mathrm{~s}$, and the TT- and $\mathrm{ZZ}$-CCFs for $\mathrm{RPM}=32$ show the upgoing $\mathrm{S}$ wave at a lag time of $1.8 \mathrm{~s}$. This means that the upgoing $\mathrm{S}$ wave and downgoing $\mathrm{P}$ wave are dominant in the ambient noise 
wavefield during the drilling and non-drilling period, respectively.

\section{Correction on the amplification of seafloor records}

Seismic waves observed at the seafloor are amplified by soft materials in the marine sediments at shallow depths (Yabe et al. 2015). The amplified amplitudes at the OBS are corrected by zero or weak amplification of those observed at the borehole sensor. We used four deep earthquakes associated with the Pacific Plate subducting beneath the Japanese Island (Table 1). The epicenters of these events are located within $200 \mathrm{~km}$ from the drilling site, which ensures near-vertical incidence of $P$ wave into the OBSs. $W_{i j}$ is the root mean squared amplitude (RMS) of the vertical component for the $i$-th event and the $j$-th station, and is given by

$$
W_{i j}=\frac{\sqrt{w_{i j}^{2}(t)}}{\sqrt{w_{i 0}^{2}(t)}},
$$

where $w_{i j}$ and $w_{i 0}$ are the vertical components at an OBS and borehole sensor, respectively, for a time window from -1 to $9 \mathrm{~s}$ from the $\mathrm{P}$ arrival time. The RMS of the horizontal component, $H_{i j}$, is defined as

$$
H_{i j}=\frac{\sqrt{u_{i j}^{2}(t)+v_{i j}^{2}(t)}}{\sqrt{u_{i 0}^{2}(t)+v_{i 0}^{2}(t)}},
$$

where $u_{i j}, v_{i j}$ and $u_{i 0}, v_{i 0}$ are the two horizontal components at an OBS and borehole sensor, respectively. Using these at a frequency band of 5-15 Hz, the site amplification factors for the vertical and horizontal components at the $j$-th station can be written as

$$
W_{j}=\frac{\sum_{i=1}^{4} W_{i j}}{4}, H_{j}=\frac{\sum_{i=1}^{4} H_{i j}}{4} .
$$

The estimated values for each of these variables are summarized in Table 2. The estimated values for different events were stable, which led to small errors. Although the amplification factors in the horizontal component are larger than those in the vertical component, such large values were also obtained previously in this region (Kubo et al. 2018; Yabe et al. 2019). Moreover, based on this correction, in the "Discussion" section, we do not incorporate sediment layers in numerical simulations when comparing the observed and calculated amplitudes.

\section{Ps reflections from inline airgun shots within $\pm 10 \mathrm{~km}$}

Research Vessel (R/V) Kaimei (hereafter Kaimei) performed successive airgun shots along the NNW-SSE trending line within $\pm 10 \mathrm{~km}$ from the drilling site (Fig. 3). Figure $3 \mathrm{~b}-\mathrm{d}$ display the three-component seismic records, where two $P$-to-s $(P S)$ reflected waves can be clearly seen in the two horizontal components. When a gently dipping interface, i.e., a megasplay fault, is present beneath the seafloor, a reflected $P$ wave emerges at a nearly constant two-way travel time within an offset of $10 \mathrm{~km}$ (Nakanishi et al. 2008): the megasplay fault is a major out-of-sequence thrust fault splayed off the plate

\begin{tabular}{|c|c|c|c|c|c|}
\hline & Date & Latitude (o) & Longitude $\left({ }^{\circ}\right)$ & Depth (km) & Magnitude \\
\hline Equation 1 & 2019/03/16 11:07:11 & 32.5795 & 136.9545 & 458.0 & 3.2 \\
\hline Equation 2 & 2019/03/16 20:16:16 & 33.0767 & 138.3772 & 365.0 & 3.3 \\
\hline Equation 3 & 2019/03/20 04:17:30 & 34.1690 & 136.6558 & 359.9 & 3.0 \\
\hline Equation 4 & 2019/03/22 17:00:18 & 33.6270 & 137.2815 & 368.0 & 3.3 \\
\hline
\end{tabular}
interface. When the offset is small, a $P s$ wave can also be

Table 1 Deep earthquakes used for estimating site amplification

\begin{tabular}{|c|c|c|c|c|}
\hline & $\begin{array}{l}\text { Amplification of the vertical } \\
\text { component }\end{array}$ & $\begin{array}{l}\text { Error for the vertical } \\
\text { amplification }\end{array}$ & $\begin{array}{l}\text { Amplification of the horizontal } \\
\text { component }\end{array}$ & $\begin{array}{l}\text { Error for the } \\
\text { horizontal } \\
\text { amplification }\end{array}$ \\
\hline OBSO1 & 1.296 & 0.136 & 14.944 & 1.247 \\
\hline $\mathrm{OBSO2}$ & 1.343 & 0.157 & 18.698 & 0.881 \\
\hline $\mathrm{OBSO3}$ & 1.476 & 0.118 & 19.446 & 0.777 \\
\hline OBSO4 & 1.406 & 0.157 & 19.595 & 1.170 \\
\hline OBS05 & 1.126 & 0.180 & 14.791 & 1.189 \\
\hline OBSO6 & 1.300 & 0.195 & 16.214 & 1.046 \\
\hline
\end{tabular}

Table 2 Estimated site amplifications at each OBSs 

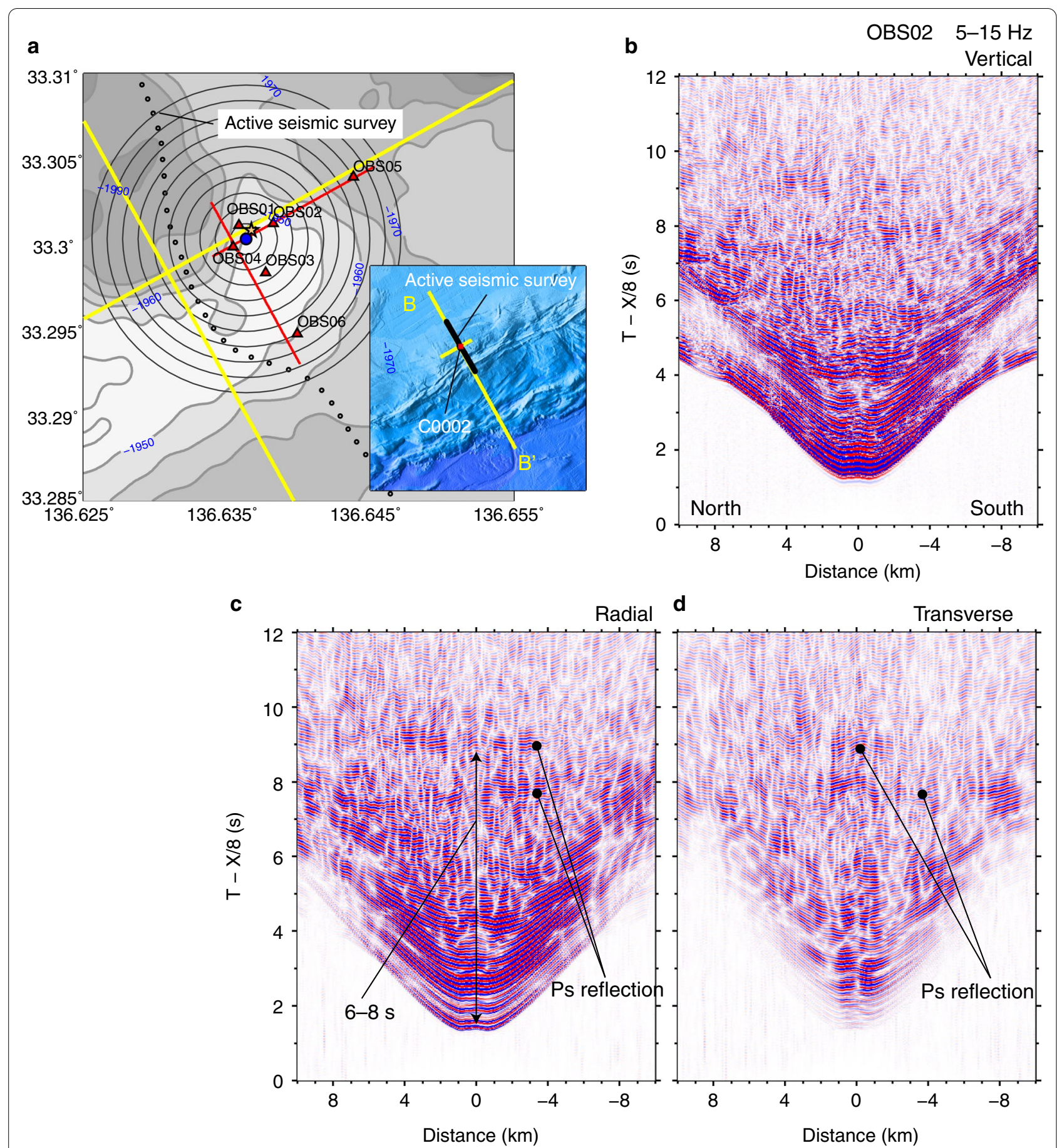

Fig. 3 Airgun shot experiment from R/N Kaimei. a Map showing the locations of inline airgun shots from R/N Kaimei (black dots). All other symbols are as in Fig. 1. b Three-component shot records observed at OBSO2 at frequency bands $5-15 \mathrm{~Hz}$. Left and right sides of the panels represent the NNW and SSE directions

observed in a similar manner. Based on $P s-P$ times of 6.1 and $7.6 \mathrm{~s}$, a $V p$ model (Shiraishi et al. 2019), and an empirical relation between $V p$ and $V s$ (Brocher 2005), two $P S$ waves are reflected from the megasplay fault at a depth of $7 \mathrm{~km}$ and the top of the oceanic crust at a depth of $9 \mathrm{~km}$. For example, using a $V p$ of $3.70 \mathrm{~km} \mathrm{~s}^{-1}$ between the megasplay fault and the top of the oceanic crust (Shiraishi et al. 2019), we obtain a $V s$ of $2.02 \mathrm{~km} \mathrm{~s}^{-1}$ from the 
empirical relation (Brocher 2005). Using a differential travel time of $1.5 \mathrm{~s}$, we obtained a thickness of $1.96 \mathrm{~km}$ between the two reflections.

\section{Ps reflections from airgun shot and ship noise by Chikyu} Although the inline-shot records from the Kaimei are useful for detecting $P s$ signals, fixed-point airgun shots from the Chikyu are more effective to compare with $P s$ signals retrieved from the Chikyu ship noise, because the ship noise and airgun shots are generated from almost the same location, whereas the location of the Kaimei was at least $400 \mathrm{~m}$ away from the Chikyu.

To retrieve Ps signals from airgun shot and ship noise, we applied a deconvolution technique in the frequency domain (e.g., Langston 1979; Ammon 1991) to the three-component records with a bandpass filter of 9-15 Hz: the horizontal component is divided by the vertical component in the frequency domain. Because we divided the deconvolved waveform by the maximum amplitude of the auto correlation of the vertical component (Ammon 1991), the absolute Ps amplitudes are preserved irrespective of different OBSs and the temporally varying strengths of ship noise. Prior to the deconvolution, waveform amplitudes in the vertical and horizontal components were divided by the site amplification factors in Table 2. The time windows for airgun and ship noise (ambient noise) records were $20 \mathrm{~s}$ from the shot origin time and $80 \mathrm{~s}$, respectively. For ship noise, hourly and daily waveforms in the radial and transverse components were obtained by stacking $80 \mathrm{~s}$-deconvolved waveforms. Here, we only used 1-h waveforms when the stacked number of the $80 \mathrm{~s}$-deconvolved waveforms with $R P M=0$ was $>40$ among 45 segments $(\times 80 s=1 \mathrm{~h})$. This processing allows us to use the continuous hourly records in which non-drilling periods are dominant.

In Figs. 4 and S2, the deconvolved waveforms from 20 airgun shots were aligned at each OBS, while the daily waveforms from ambient noise on Julian days 1-120 of 2019 were aligned, in which the Chikyu was located onsite on Julian days 1-58 and was away from the site after Julian day 59: this indicates that none of the signals after Julian day 59 were caused by no ship noises. In these figures, we focused on lag times of 4-9 s by referring to $P s-P$ times of $6.1 \mathrm{~s}$ and $7.6 \mathrm{~s}$ at the megasplay fault and the top of the oceanic crust. Non-drilling periods $(\mathrm{RPM}=0)$ were relatively secured during days $1-58$ in 2019 compared with the project period in 2018 (Fig. 2b), and the location of the Chikyu was ranged within $\pm 5 \mathrm{~m}$ and mostly $\pm 1 \mathrm{~m}$ (the Chikyu was located within a radius of $1 \mathrm{~m}$ for $90.06 \%$ of the days 1-58) during the study period (Figs. 2c, S3a, and S3b). Moreover, in order to evaluate the similarity of the deconvolved waveforms, we estimated cross-correlation coefficients (CCs) between the deconvolved waveforms using airgun shot and ship noise records, with a time window of $0.1 \mathrm{~s}$ and a time shift of $0.05 \mathrm{~s}$ (Figs. 4 and S2).

Coherent signals between the two sources emerged at lag times of 5, 6, and $7.8 \mathrm{~s}$ in the radial-deconvolved waveforms (RDWs) at OBS03 (Fig. 4a), but signals were clearer in the transverse component and could be observed at all OBSs (Fig. 4b and S2). Although coherent signals appear in either source, e.g., $4.3 \mathrm{~s}$ in the ship noise (Fig. 4a, b), this may be caused by a volumetric source difference between the ship noise and airgun shot. Whereas the airgun shot is excited by a point source, the ship noise is generated by a small volumetric source due to the internal instrumental and external thruster noises. Such a volumetric difference may have a different sensitivity to reflectors, and result in a difference of $P s$ extraction in the deconvolved waveforms using the ship noise and airgun shots. Also, the RDWs contain multiple peaks compared with the TDWs (Fig. 4). Those peaks may have originated from the contamination of multiple reflections at shallow depth. In theory, the reflection coefficient of $P$-to- $S$ reflection from a horizontal boundary for a vertical incident angle is zero. However, when the incident angle of the $P$ wave and the boundary are slightly deviated from the vertical direction and horizontal plane, the reflection and its multiple reflections are possibly observed in the RDWs at smaller lag times and these are deviated to other directions at larger lag times. Moreover, we estimated the CCs of the RDWs and TDWs between airgun shots and ship noises, and coherent signals were obtained with higher CCs in the TDWs (Fig. 4d). We therefore avoided the use of the RDWs in the subsequent processing. Although all the signals in the TDWs obtained from the two sources did not match completely, it is likely that the coherent signals originated from the

\footnotetext{
(See figure on next page.)

Fig. 4 Radial- and transverse-deconvolved waveforms (RDWs and TDWs) for DN Chikyu airgun and ship noise. a RDWs for DN Chikyu airgun (left) with 20 shots and ship noise (right) during Julian days 1-58 at OBS03. The days without data (gray shaded areas) correspond to the drilling period, and quiet data after day 59 correspond to the period when D/N Chikyu moved to other sites. Arrows represent coherent signals that can be observed in both the RDWs. b Same as panel a, but for TDWs. c (top) Waveforms stacked over (black) 20 shots and (red) 58 days in panel a. (bottom) Cross-correlation coefficients between the red and black waveforms in the top panel, with a time window of $0.1 \mathrm{~s}$ and a time shift of $0.05 \mathrm{~s}$. $\mathbf{d}$ Same as c, but for panel b. e Same as panel a, but for OBS02. f Same as panel a, but for OBS05
} 


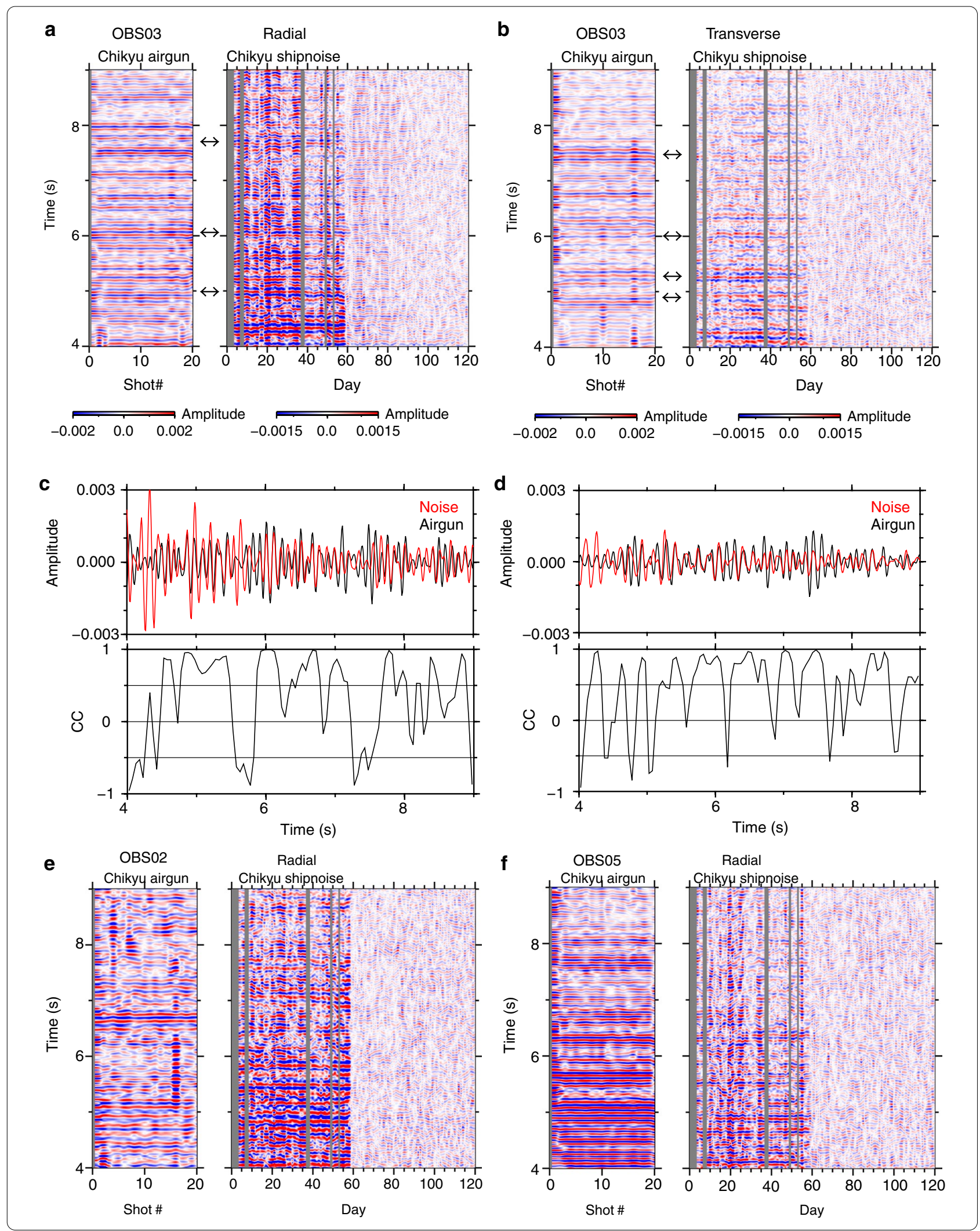


same structural boundaries beneath the seafloor, including the megasplay fault, because peaks with high $C C$ values could be observed in the TDWs between the airgun shots and ship noises (Additional file 1: Fig. S2).

\section{Stacking waveforms with tidal stress}

The tidal normal stress on the megasplay fault beneath the Chikyu was calculated with a method described in a previous study (Yabe et al. 2015), in which the tidal stress tensor on the plate interface associated with the both body tide and the ocean tide was calculated, and the stress tensor was converted to traction (normal stress) on the fault using a dip, strike, and rake on the plate interface. The geometry of the megasplay fault was inferred with a strike $=270^{\circ}$ and dip $=10^{\circ}$, which were set by referring to three-dimensional reflections and velocity models in this region (Shiraishi et al. 2019). The slip direction had a rake $=125^{\circ}$, which represents the thrust fault. The azimuth and angle were defined as clockwise from north and downward from the horizontal plane. Figure 5 a shows the resulting normal stress on the megasplay fault, in which negative values represent compression. We stacked 1-h TDWs at each 5 days for the following three ranges in normal stress at every OBS: high tide $<-2.4 \mathrm{kPa}$, middle tide between -2.4 and $1.6 \mathrm{kPa}$, and low tide $>1.6 \mathrm{kPa}$ (Fig. 5a). The envelope functions were calculated by stacking 5-day TDWs during 10-50 Julian days when the number of available TDWs were balanced among the three tide ranges (Figs. 6 and S4): during the 5 days, $120 \mathrm{~h}$ (maximum) were divided by the three ranges of the tide, and we counted the numbers of 1-h TDWs in the three ranges (Fig. 5b). The above thresholds for the three ranges of normal stress were determined so as to preserve the number of $1-\mathrm{h}$ TDWs to at least 5 during every 5 -day period and for each range. Although the resulting counts on the 37th day were less due to the drilling periods (Fig. 2b) and the count for middle tide on the date was 5 , those for high and low tides exceeded 9 (Fig. 5b). We therefore compared the TDWs between high and low tides in this study. The uncertainties of the envelope functions were the standard deviations obtained by bootstrapping the 5-day TDWs for each tide range for 5,000 times with repetition (Figs. 6 and S4).

To investigate the stability of the amplitudes in the stacked TDWs, we plotted 5-day moving averages of the TDWs for the normal stress ranges of high and low tides (Fig. 5c, d). Moreover, the delay time of each segment in the 5-day TDWs between high and low tides was also estimated by cross-correlating them with a time window of $0.2 \mathrm{~s}$ and a time shift of $0.1 \mathrm{~s}$ (Fig. 5c, d). If some signals in the TDWs were systematically delayed (or advanced) between high and low tides, the delay time should have showed the coherent patterns at similar lag times.

\section{Migration $P$-to-s scattered amplitude and tidal-responded amplitude}

Using the three-dimensional $V p$ model (Shiraishi et al. 2019) and an empirical relation between $V p$ and $V s$ (Brocher 2005), we calculated differential travel times between direct $P$ waves from the Chikyu to an OBS and $P$-to-s $(P s)$ scattered waves in two-dimensional transects. The amplitudes of the TDWs were plotted onto locations where their lag times were matched with the differential times in the transects. Here, the TDWs were stacked over 10-50 days at every OBS, and the amplitudes in the stacked TDWs were assumed to have been due to $P s$ waves. The TDW amplitudes at lag times of 5-9 s were used for imaging, where $P s$ waves reflected around the plate boundary were expected. We only imaged below OBSs within a scattering angle of the $P s$ wave less than $2^{\circ}$ from the vertical axis, because the number of OBSs was small for imaging the entire volume around the OBS array. The scattering angle was defined as the angle between the incident $P$ wave and the $P s$ wave at scattering points.

In addition to the raw amplitudes, we also created transects for displaying the locations where amplitudes responded to tidal stress. To enhance the amplitude variations in response to tidal forces, we calculated the amplified differential $P s$ amplitudes (AD-Ps) between the envelope functions of the high-tide TDW, $S_{H}(t)$, and low-tide TDW, $S_{L}(t)$,

$$
F(t)=\frac{R(t)}{|R(t)|} S_{H}(t) \cdot R(t)^{2} \cdot C,
$$

where

$$
R(t)=S_{H}(t)-S_{L}(t),
$$

and $C$ is an arbitrary constant which only makes the average amplitude of $F(t)$ comparable to that of $S_{H}(t)$. We chose $C$ as $2.0 \times 10^{6} . R(t)$ indicates the difference of $P s$ amplitudes in the envelope functions of $S_{H}(t)$ and $S_{L}(t)$ (Eq. (5)). The positive/negative polarity in $R(t)$ represents the velocity contrast increase/decrease at seismic discontinuities at high tide. When the large $P s$ amplitude in $S_{H}(t)$ and large $P s$ amplitude difference in $R(t)$ occur at the same lag times, the peak amplitudes in the $S_{H}(t)$ were amplified while preserving the polarities of $R(t)$. Thus, $F(t)$ shows $P s$ reflections from seismic discontinuities with large impedance contrasts and large amplitude variations between high and low tides. The examples of $S_{H}(t)$ and $F(t)$ at OBS03 and OBS06 are displayed in Figs. 6b and S4b. Using these functions, we made ENE-WSW and 

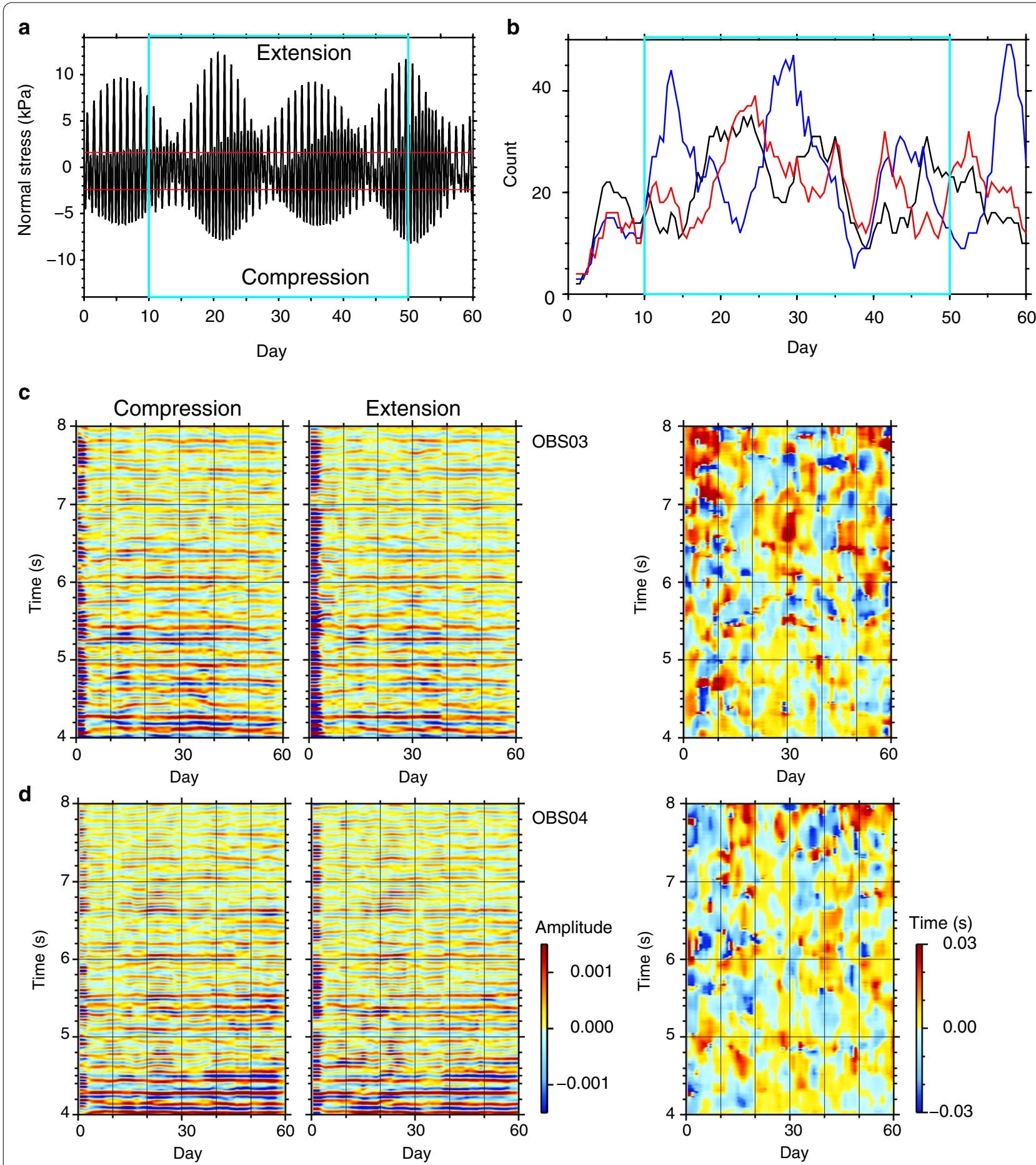

Fig. 5 Tidal response of TDWs. a Normal stress on the megasplay fault, calculated from the method described by Yabe et al. (2015) (Yabe et al. 2015). Normal stress values within the light-blue rectangle were used to calculate the stacked envelope function in Fig. 6 . The red lines represent the thresholds at high and low tides. b Number of stacked 1-h TDWs within 5-day moving averages at (black line) high tide, (blue line) middle tide, and (red line) low tide. c The first two panels on the left represent 5-day stacked TDWs during Julian days 1-60 in 2019 for high and low tides at OBS03. The frequency band is $9-15 \mathrm{~Hz}$. The right panel displays the time shift of each segment between the high and low tides, and the time window of the segment and increment are 0.2 and 0.1 s, respectively. $\mathbf{d}$ Same as panel c, but for OBSO4 


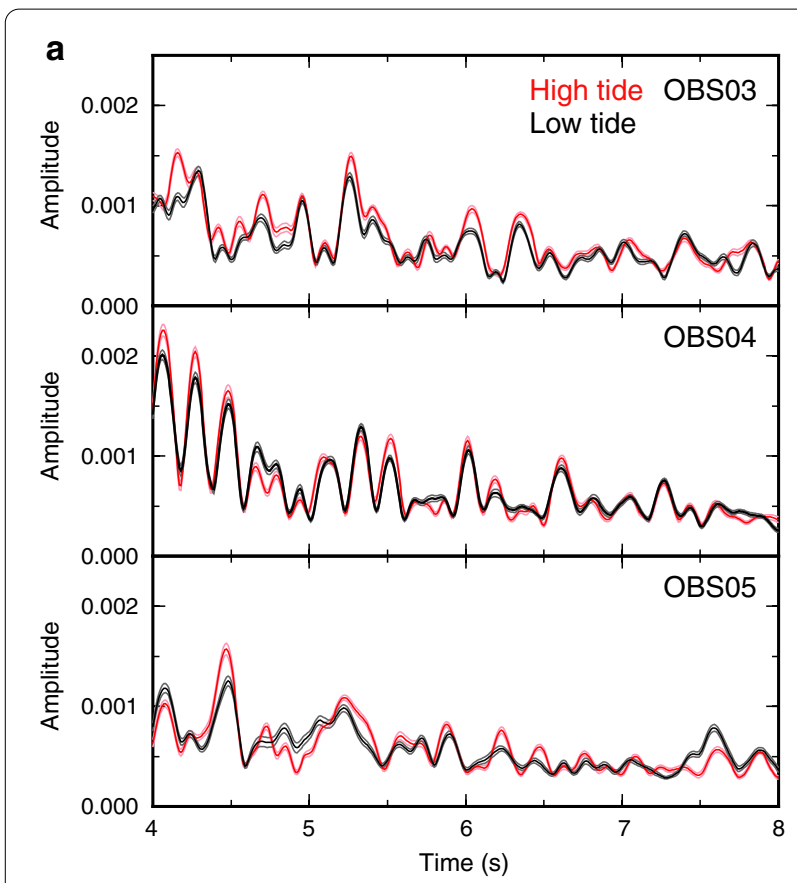

b

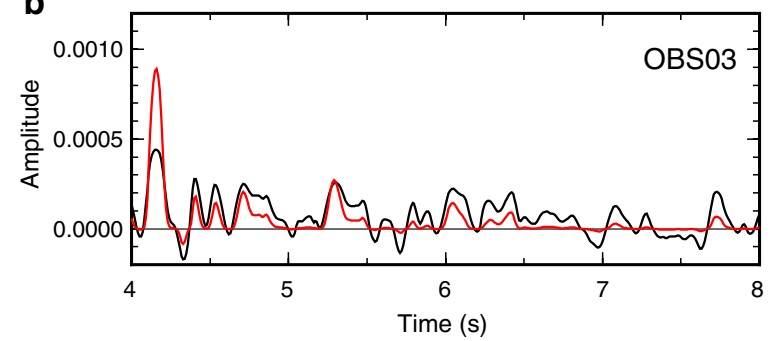

Fig. 6 Examples of transverse-deconvolved waveforms (TDWs) for different tidal stress. a The red and black lines represent the TDWs at high and low tide, respectively, for three OBSs. The standard deviations are represented by pink and gray lines at high and low tides, respectively, for OBS03. $\mathbf{b}$ The black line shows the differential amplitude of the envelope TDWs stacked over 10-50 days in 2019 between high tide and low tide at OBS03. The red line shows the amplified amplitudes of the black line, derived using Eq. (4)

NNW-SSE trending transects (red lines in Fig. 1b) and projected them onto the reflection images (Shiraishi et al. 2019) along nearby ENE-WSW and NNW-SSE trending lines (yellow lines in Fig. 1a, b). Because AD-Ps at OBS01 was slightly large compared with that at other OBSs and the amplitudes in the TDWs may have been unstable during 1-58 Julian days (Additional file 1: Fig. S2a), we removed this station from imaging.

\section{Results}

\section{Tidal response of reflections}

To investigate whether the amplitudes and travel times of $P s$ waves respond to tidal stress, we calculated the normal tidal stress on the megasplay fault (Yabe et al. 2015) by

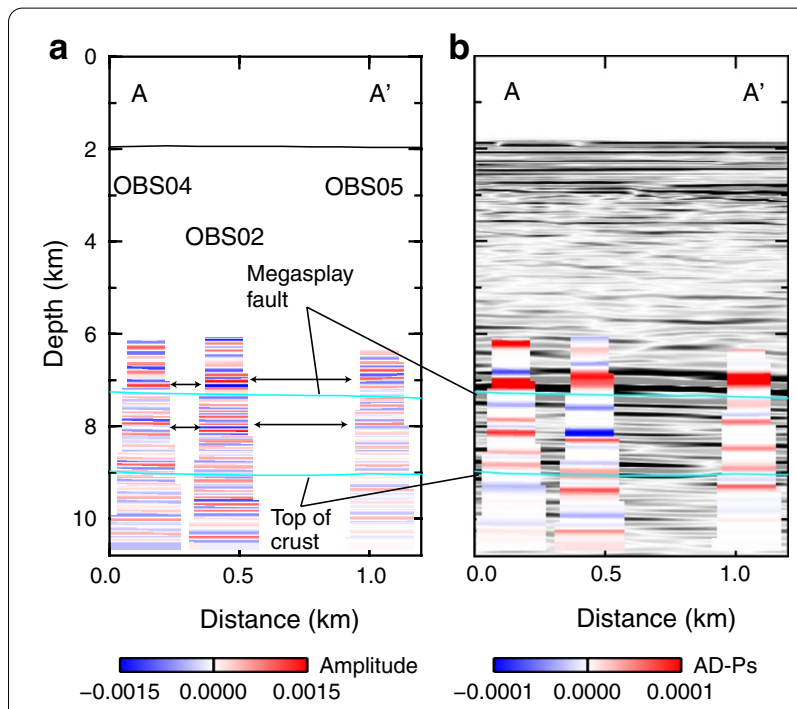

Fig. 7 Migration image of Ps wave projected onto the reflection image. a Migration image of Ps waves in the TDWs along $A-A^{\prime}$. The light-blue lines show the geometries of megasplay fault and the top of the oceanic crust (Shiraishi et al. 2019). The arrows indicate large Ps amplitudes, which correspond to the megasplay fault and a fault between the megasplay fault and the top of the oceanic crust. $\mathbf{b}$ Migration image of AD-Ps of (a) projected onto the reflection image along the ENE-WSW trending yellow line in Fig. 1b (Shiraishi et al. 2019)

assuming its geometry (Shiraishi et al. 2019) and thrust fault slip derived from the motion vector of the underlying ocean plate (DeMets et al. 2010) (Fig. 5a). Figure 6 presents the comparison of envelope TDWs using ship noise at high and low tide, mainly showing larger peak amplitudes of $P s$ waves at high tide. This feature can be partially recognized at OBS02 and OBS06 (Additional file 1: Fig. S4), although some peaks show smaller amplitudes at high tide. The stability of the larger amplitudes at high tide at $\mathrm{OBS} 03$ and OBS04 were also confirmed by the 5-day moving average of the TDWs (Fig. 5). In addition, the time-shift estimation of waveform segments of the TDWs between high and low tides with a time window of $0.2 \mathrm{~s}$ and a time increment of $0.1 \mathrm{~s}$ did not vary systematically (Fig. 5c, d). These observations indicate that seismic velocity changes in the entire medium associated with tidal stresses are minor, whereas velocity contrasts at the boundaries within the accretionary prism fluctuated with tidal stress.

\section{Imaging of tidal responding boundaries}

Depth migrations of amplified differential Ps amplitudes (AD-Ps) between the high and low tides (Eq. (4)) located where the velocity contrast fluctuates in the accretionary prism. The Ps signals of the TDWs and the AD-Ps were strong at the megasplay fault at a depth of $7 \mathrm{~km}$, whereas 
those signals were weak at the top of the oceanic crust at a depth of $9 \mathrm{~km}$ (Fig. 7). Here, for AD-Ps, a positive/ negative amplitude represents a correspondingly positive/negative enhancement of $P s$ amplitude at high tide, respectively, and the AD-Ps at three stations close to the line are plotted. Additionally, the velocity contrast of a boundary at a depth of $8 \mathrm{~km}$ between the megasplay fault and the top of the oceanic crust fluctuates with tidal stress. More of the clear features of the two boundaries can be recognized in the NS-trending transect (Fig. 8).

\section{Discussion}

\section{Velocity reduction in isotropic case}

A seismic exploration survey revealed the negative amplitude of $P$ waves reflected at the megasplay fault (Park et al. 2002), which is indicative of a velocity reduction within the fault. The larger positive amplitude observed at high tide in this study indicates that the velocity reduction at the megasplay fault was further enhanced by vertical compression caused by tidal stress.

To confirm the velocity reduction, we conducted a three-dimensional finite-difference approach with rotated staggered grids and second-order calculation accuracies in time and space (Saenger et al. 2000). The model space $\left(x_{1}-x_{2}-x_{3}\right)$ was $8 \times 8 \times 6 \mathrm{~km}^{3}$ and included four layers with a grid spacing of $0.01 \mathrm{~km}$, and the $x_{3}$-axis was taken as vertically downward. The time step was $0.001 \mathrm{~s}$. An absorbing boundary condition was assigned to each side except for the top (Clayton and Engquist 1977). The source location was set to $\left(x_{1}, x_{2}, x_{3}\right)=(4.0,4.0,0.0)$ at the model sea surface. A vertical force with a Ricker wavelet at a center frequency of $7.59 \mathrm{~Hz}$ (maximum frequency of $10.96 \mathrm{~Hz}$ ) was applied to the source location. The stations were assigned at the seafloor locations $(2 \mathrm{~km}$ depth) relative to the Chikyu in the observations (Fig. 9a). The $V p, V s$, and density of the homogeneous medium and fluid are $4.5 \mathrm{~km} \mathrm{~s}^{-1}, 2.25 \mathrm{~km} \mathrm{~s}^{-1}$, and $2.5 \mathrm{~g} \mathrm{~cm}^{-3}$, and $1.5 \mathrm{~km} \mathrm{~s}^{-1}, 0.0 \mathrm{~km} \mathrm{~s}^{-1}$, and $1.0 \mathrm{~g} \mathrm{~cm}^{-3}$, respectively (Table 3), in which $V p$ is roughly referred to an averaged $V p$ around the megasplay fault. In the isotropic case, a $V s$ of $2.25 \mathrm{~km} \mathrm{~s}^{-1}$ at layer 3 was reduced by $1,3,7$, and $13 \%$ (Table 4). The fault thickness was set to be $0.05 \mathrm{~km}$ (Rowe et al. 2013), in which $V p$ and $V p / V s$ were referenced to seismic exploration results (Shiraishi et al. 2019).

Prior to comparing the observed amplitude difference (0.0002 in Fig. 6a), and the results of the numerical simulation, we confirm the stability of the TDW amplitudes that are possibly affected by the location of the Chikyu. The position of the Chikyu was measured at each minute. We divided the Chikyu positions into eight regions, during 1-58 Julian days in 2019 (four quadrants, each with inside and outside $1 \mathrm{~m}$ from the central point of the positions) (Additional file 1: Fig. S3a), and stacked TDWs at OBS03 depending on the region in which the Chikyu was located (Additional file 1: Fig. S3c). Because the megasplay fault is dipping, the stacked TDW amplitudes may be varied by the location of the Chikyu (4 quadrants). For the stacked TDWs, we calculated the standard deviation of the amplitudes at a time window of 5-9 $\mathrm{s}$ in the lag time among nearby regions A-D (Additional file 1: Fig. S3c top) and among distant regions $\mathrm{E}-\mathrm{H}$ (Additional file 1: Fig. S3c bottom), and obtained standard deviations of $6.49 \mathrm{e}-5(0.0000649)$ and $2.28 \mathrm{e}-4(0.0002283)$, respectively. The former value indicates that, if the Chikyu is located within $1 \mathrm{~m}$ from the central point of the positions, the observed amplitude difference between the high and low tides is significantly larger than the amplitude variation due to the location changes, and indeed the Chikyu was almost located within a radius of $1 \mathrm{~m}$ during 1-58 Julian day (90.06\%). The latter value is comparable to the observed difference, but this may be caused by
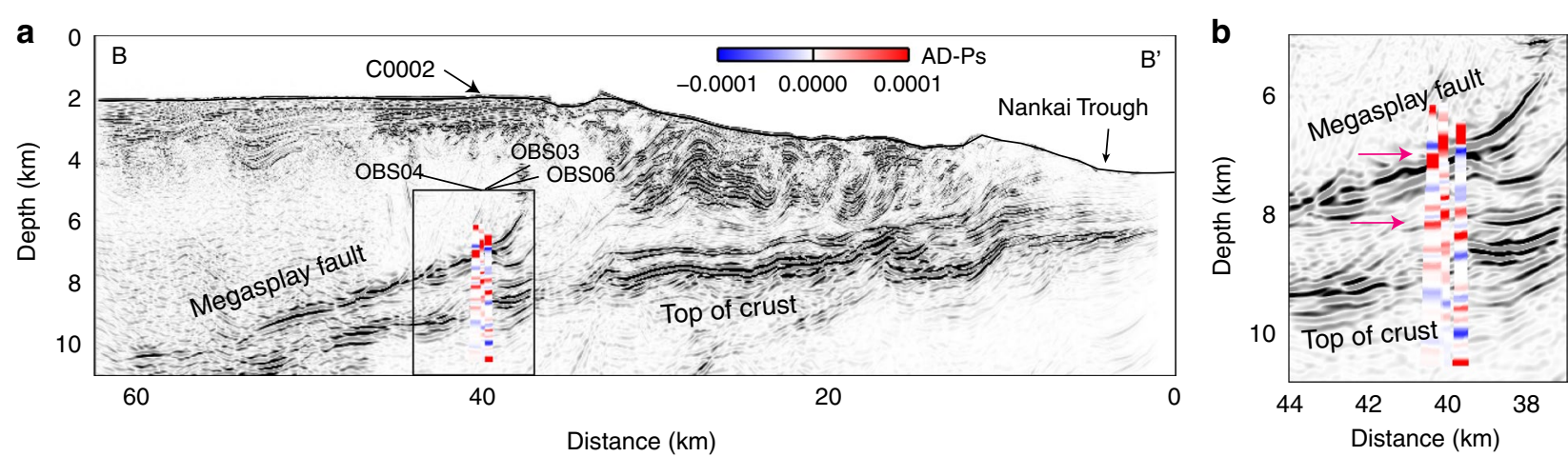

Fig. 8 Migration images of AD-Ps projected onto the reflection images. a Migration image of amplified differential Ps amplitudes between high and low tide (Eq. (4)) in the TDWs projected onto the reflection image along B-B' (Fig. 1) (Shiraishi et al. 2019). b Zoom in on the box in panel a. The red arrows indicate multiple weak interfaces, including the megasplay fault 
a

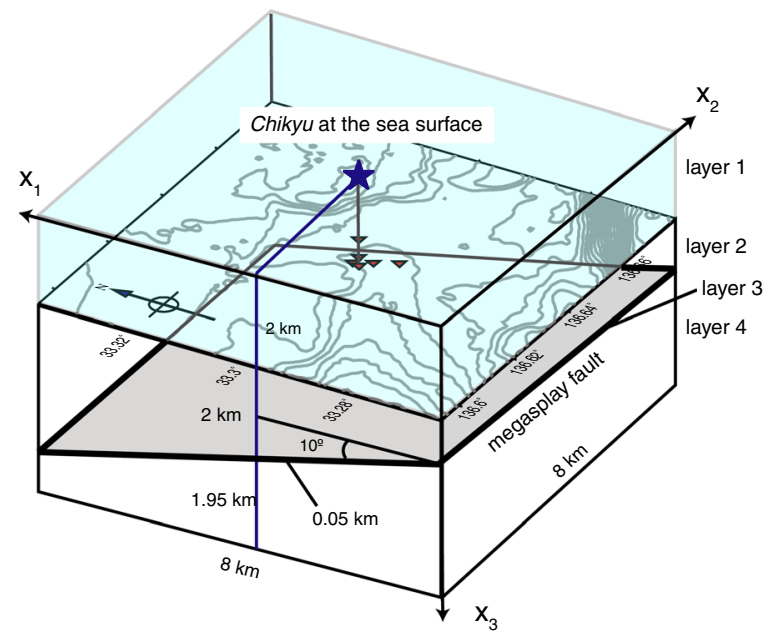

b

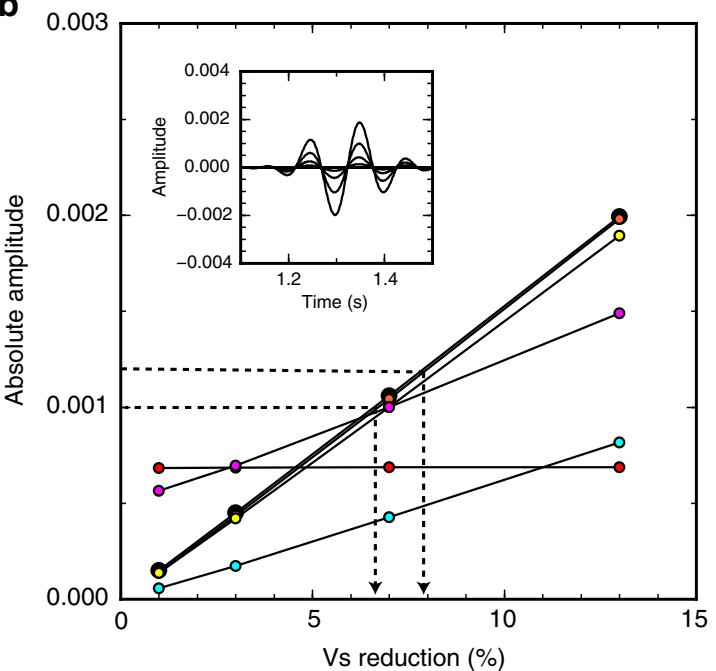

Fig. 9 Numerical simulations for Ps reflected waves. a The source location of DN Chikyu was set at the sea surface. Bathymetry is flat. The red triangles represent the six OBSs, whose relative horizontal locations with respect to D/N Chikyu were preserved. The physical properties at each layer are summarized in Tables 3, 4, 5. b Ps amplitudes reflected at layer 3 as a function of Vs reductions in Table 3 for the six OBSs, yellow: OBS01, black: OBS02, light-blue: OBS03, orange: OBS04, red: OBS05, and magenta: OBS06. The two dashed arrows show the Vs reduction at OBS04 for an amplitude variation of 0.0002

Table 3 Model space and physical parameters for numerical simulation

\begin{tabular}{|c|c|c|c|c|c|c|}
\hline & Thickness (km) & $V p\left(\mathrm{~km} \mathrm{~s}^{-1}\right)$ & $V_{s}\left(\mathrm{~km} \mathrm{~s}^{-1}\right)$ & Density $\left(\mathrm{g} \mathrm{cm}^{-3}\right)$ & $\operatorname{Dip}\left({ }^{\circ}\right)$ & Strike $\left(^{\circ}\right)$ \\
\hline Layer 1 & 2 & 1.5 & 0 & 1 & & \\
\hline Layer 2 & 2 & 4.5 & 2.25 & 2.46 & & \\
\hline Layer 3 & 0.05 & * & * & 2.46 & 10 & 270 \\
\hline Layer 4 & 1.95 & 4.5 & 2.25 & 2.46 & & \\
\hline
\end{tabular}

*This value is listed in Table 4

Table 4 Model space and physical parameters for numerical simulation

\begin{tabular}{lllll}
\hline & Thickness $(\mathrm{km})$ & $V_{p}\left(\mathrm{~km} \mathrm{~s}^{-1}\right)$ & $V_{s}\left(\mathrm{~km} \mathrm{~s}^{-1}\right)$ & Density $\left(\mathrm{g} \mathrm{cm}^{-3}\right)$ \\
\hline Layer 1 & 2 & 1.5 & 0 & 1 \\
Layer 2 & 2 & 4.5 & 2.25 & 2.46 \\
Layer 3 & 0.05 & 4.5 & $2.1375(5 \%)$ & 2.46 \\
& & & $2.0250(10 \%)$ & \\
& & & $1.9125(15 \%)$ & \\
Layer 4 & 1.95 & 4.5 & $2.2500(20 \%)$ & 2.46 \\
\hline
\end{tabular}

both the position fluctuations of the Chikyu and the lack of stacked TDWs.

Comparing the observed and calculated amplitudes, because the $P S$ amplitude difference is linear with respect to $V s$ reduction, the observed amplitude difference (0.0002 in Fig. 6a) can be linked to a $V s$ reduction of $1.5-3.3 \%$ (Fig. 9b). In this calculation, Ps signals were not observed in the transverse component at OBS05 and OBS06. The Ps amplitudes at these stations obtained in our observation may have been produced by a focusing effect of $P s$ amplitudes at a gently curved interface of the megasplay fault that was imaged by a 3D seismic volume (Shiraishi et al. 2019), and so these were not used to estimate $V s$ reductions. Because the obtained $V s$ reduction of $1.5-3.3 \%$ is large and elastic property perturbations linearly changed by the tidal stress are small, it is therefore necessary to consider other mechanisms capable of significantly changing the seismic velocity of the fault with small stress fluctuations and fluid concentrations.

\section{Fracture connection and isolation due to tidal stress}

A possible mechanism is fracture channeling with fluids in the fault by external forces (e.g., Hawthorne and Rubin 2010; Houston 2015). Characteristic fractures are formed around a through-going fault ( $Y$ shear) (Bartlett 

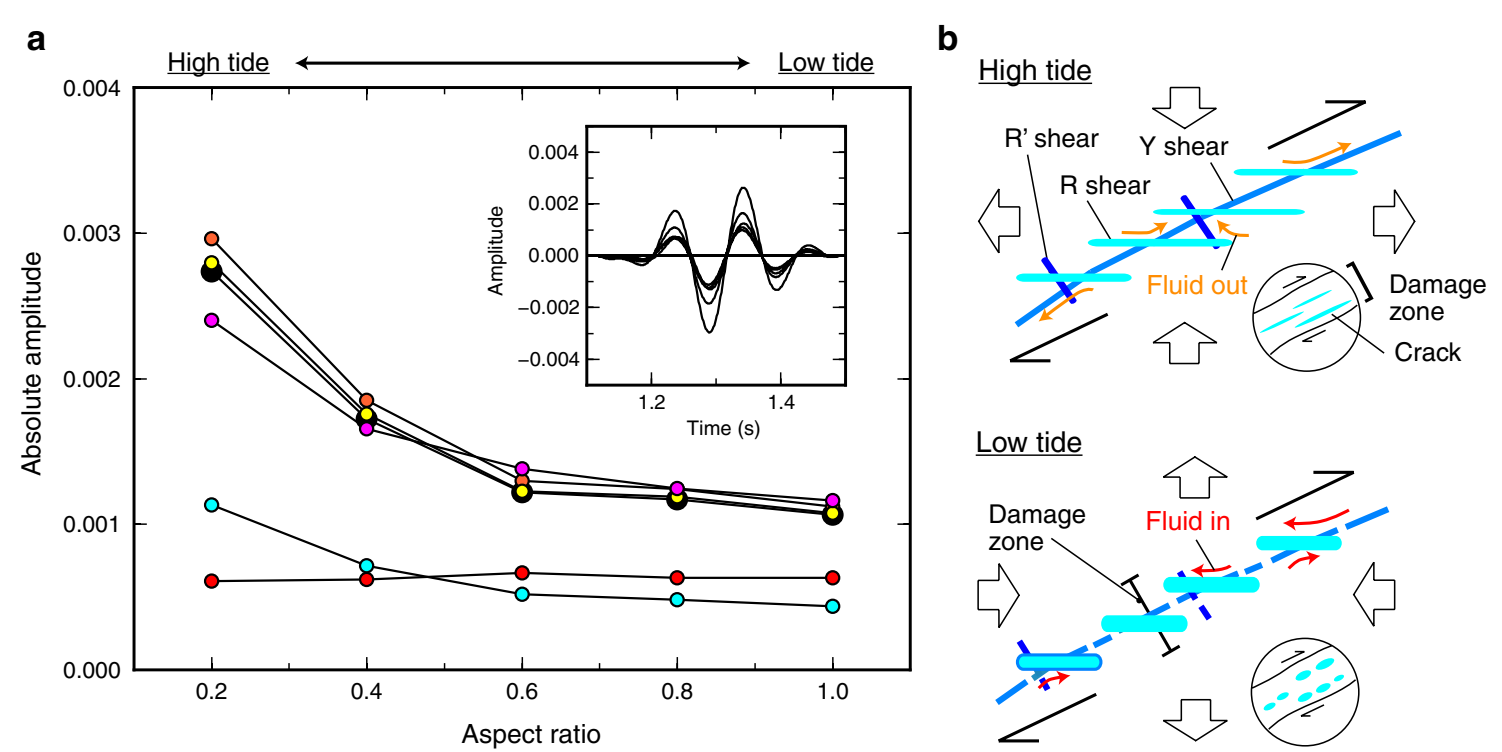

Fig. 10 Numerical simulation for the Ps amplitude reflected at the megasplay fault containing different aspect ratios of cracks. a The absolute amplitudes of Ps reflection in the synthetic TDWs for the 6 OBSs, yellow: OBS01, black: OBS02, light-blue: OBS03, orange: OBS04, red: OBS05, and magenta: OBS06. The inset displays the synthetic TDW waveforms at OBS04, where large amplitudes were obtained at low aspect ratio. $\mathbf{b}$ Sketches and circled insets show connected/less-connected fractures and their associated crack geometry at high tide/low tide (macroscopic view), respectively. Arrows of "fluid in" and "fluid out" indicate fluid migration from $R$ shears

et al. 1981) within the damage zone of a fault, with different direction groups including, e.g., Riedel $(R)$ shears and antithetic $R$ ' shears (Tchalenko and Ambraseys 1970). On land, these shear zones are found in the latest Cretaceous Mugi mélange in southwestern Japan, and this mélange is considered to be a fault rock along the plate boundary in the Nankai subduction zone (Kimura et al. 2012). These fractures around the deep plate boundary are potentially saturated with fluids, because the region between the fault and top of oceanic crust is characterized by high pore pressures (Tsuji et al. 2014), and the land mélange also shows evidence of fluid presence (Kimura et al. 2012). For a dip angle of the megasplay fault of $10^{\circ}$ in this region (Shiraishi et al. 2019), the $R$ shears are oriented close to horizontal (Fig. 10). When a small vertical-compressional stress is applied to $R$ shears, their shapes are slightly compressed in the vertical direction, and internal fluids are expelled to $Y$ shear and near-vertical $R$ ' shears following the fluid pressure gradient. Here, because the porosity around the fault is 0.07 (Tsuji et al. 2008), the fault transport condition is close to the undrained one, and hence it is considered that the diffusive propagation of tidal stress variations through fluids, which can be observed at the top of the marine sediment with high porosity (Wang and Davis 1996), do not occur within the fault. Slight fluid migration results in fluid connections between shears, thereby enhancing seismic velocity anisotropy within the megasplay fault. By this mechanism, negative AD-Ps would be caused only when near-vertical $R$ ' shear expansion is prevalent and there is less connectivity of $Y$ shears in the fault-parallel direction.

\section{Numerical simulation in anisotropic case}

To qualitatively investigate large $P s$ amplitudes reflected at the megasplay fault, we also conducted numerical simulations for models in which the degree of anisotropy within the fault varied with the aspect ratio of cracks. Here, the assumptions for an effective medium in the damage zone along the megasplay fault are that highly/ weakly connected fractures are described by inclusions of fault-oriented oblate spheroidal cracks with a small/large aspect ratio, with a constant porosity of the bulk medium at 0.07 by referring to the porosity $<0.07$ of land outcrop samples that were considered to be a fossil seismogenic megasplay fault rock (Tsuji et al. 2008).

The calculation of the effective elastic constants for a medium containing fluid-filled fractures and their connections would be complicated. Instead, for the purpose of this study, we assumed a simple model in which fluidfilled oblate spheroidal cracks, assuming Riedel shears, are horizontally developed within the megasplay fault with a dip angle of $10^{\circ}$. When the cracks are partially connected by fluid-filled fractures, such as $Y$ and $R^{\prime}$ shears, due to an external force, individual fracture lengths are extended along the fault-parallel direction. This would be expressed by a slight decrease in the average aspect 
Table 5 Effective elastic constants as a function of aspect ratio of cracks

\begin{tabular}{|c|c|c|c|c|c|c|c|}
\hline Aspect ratio & $\mathrm{C} 11$ & $\mathrm{C} 12$ & $\mathrm{C} 13$ & C33 & C44 & $\begin{array}{l}V p \text { fast }\left(\mathrm{km} \mathrm{s}^{-1}\right) \\
V p \text { slow }\left(\mathrm{km} \mathrm{s}^{-1}\right)\end{array}$ & $\begin{array}{l}\text { Vs fast }\left(\mathrm{km} \mathrm{s}^{-1}\right) \\
\text { Vs slow }\left(\mathrm{km} \mathrm{s}^{-1}\right)\end{array}$ \\
\hline 1.0 & 40.310 & 19.225 & 19.201 & 40.310 & 10.389 & 4.14 & 2.10 \\
\hline \multirow[t]{2}{*}{0.8} & 40.817 & 19.454 & 19.088 & 39.197 & 10.309 & 4.17 & 2.12 \\
\hline & & & & & & 4.08 & 2.09 \\
\hline \multirow[t]{2}{*}{0.6} & 41.452 & 19.751 & 18.856 & 37.459 & 10.147 & 4.20 & 2.13 \\
\hline & & & & & & 3.99 & 2.08 \\
\hline \multirow[t]{2}{*}{0.4} & 42.249 & 20.142 & 18.351 & 34.457 & 9.791 & 4.24 & 2.15 \\
\hline & & & & & & 3.83 & 2.04 \\
\hline \multirow[t]{2}{*}{0.2} & 43.865 & 20.671 & 17.074 & 28.433 & 8.734 & 4.32 & 2.17 \\
\hline & & & & & & 3.48 & 1.93 \\
\hline
\end{tabular}

ratio of the cracks aligned to the fault-parallel direction with minor porosity changes. The $P$ wavelength used in this study is $300-500 \mathrm{~m}$ using a frequency of $9-15 \mathrm{~Hz}$ and a $V p$ of $4.5 \mathrm{~km} \mathrm{~s}^{-1}$ (Shiraishi et al. 2019), and the fault thickness at the Nankai accretionary prism toe was estimated to be $47.5 \mathrm{~m}$ from IODP Site C0007 (Rowe et al. 2013). Because the $P$ wavelength is sufficiently large compared to the fault thickness and therefore to the fracture sizes, the assumption that fracture connections can be described by the changes in the average aspect ratio of aligned cracks is acceptable.

We calculated effective elastic constants using differential effective medium (DEM) theory for hexagonal symmetry, in which aligned oblate spheroidal cracks with an aspect ratio are contained in a homogeneous medium of given porosity (0.07) (Nishizawa 1982; Tonegawa et al. 2013). The effective elastic constants transformed into those in which the axis was parallel to the normal direction of the megasplay fault with a dip angle of $10^{\circ}$ and strike $=270^{\circ}$ using the following equation (Nagaya et al. 2008).

$$
c_{i^{\prime} j^{\prime} k^{\prime} l^{\prime}}=U_{i i} U_{i j j^{\prime}} U_{k k^{\prime}} U_{l l^{\prime}} C_{i j k l},
$$

where the elastic constants $C_{i j k l}(i, j, k, l=1,2,3)$ are transformed into those $c_{i{ }^{\prime \prime} k^{\prime} l^{\prime}}$ defined in the $\left(x_{1}{ }^{\prime}-x_{2}{ }^{\prime}-x_{3}\right)$ coordinate system. $C_{I J}$ in Table 5 was converted into $C_{i j k l}$ using the relationships of $i j=11,22,33,23,13,12$ when $i$ $(=j)=1,2,3,4,5,6$ and

$$
C_{i j k l}=C_{j i k l}=C_{i j l k}=C_{k l i j} \text {. }
$$

The matrix elements $U_{i i^{\prime}}$ can be written as

$$
U=\left(\begin{array}{ccc}
\cos \xi \cos \lambda & \cos \xi \sin \lambda & -\sin \xi \\
-\sin \lambda & \cos \lambda & 0 \\
\sin \xi \cos \lambda & \sin \xi \sin \lambda & \cos \xi
\end{array}\right),
$$

where $\xi$ and $\lambda$ are the tilt angle of the symmetry axis measured from the $x_{3}$-axis and the azimuth of the axis measured clockwise from north ( $x_{1}$-axis), respectively. We set $\xi=10^{\circ}$ and $\lambda=180^{\circ}$ in the numerical simulations. The converted physical parameters were inserted in layer 3 in Table 3. In the isotropic case (aspect ratio $=1.0), V p$ and $V s$ in the layer were reduced from 4.50 to $4.25 \mathrm{~km} \mathrm{~s}^{-1}$ and from 2.25 to $2.15 \mathrm{~km} \mathrm{~s}^{-1}$, respectively, because the porosity including fluids was set to 0.07 . When the aspect ratio of cracks varied from 1.0, the fast and slow seismic velocities are slightly deviated from those values. The aspect ratios are $0.2-1.0$ with an increment of 0.2 . The calculated seismic velocities are summarized in Table 5 .

The results show that $P s$ amplitudes tend to increase as the aspect ratio of cracks decreases following a nonlinear curve (Fig. 10), except for the result at OBS05 as well as the isotropic case. Thus, our results indicate that fault-parallel connections of fractures produce large $P s$ amplitudes in our observation geometry, and that if fractures are originally moderately connected (i.e., their aspect ratio is small), significant amplitude variations can be induced by a small number of additional connections between fractures.

Moreover, we roughly estimated the preferred aspect ratio of cracks within the megasplay fault in this region using both our observation results and numerical simulations. The observed Ps amplitude and its difference between high and low tides were $\sim 0.001$ and $\sim 0.0002$, respectively (Fig. 6). These values indicate that the aspect ratio of cracks is close to $0.8-1.0$, and that it varies within $0.6-1.0$ between high and low tides (Fig. 10). However, if the porosity within the megasplay fault was smaller than 0.07, e.g., 0.05 in Fig. 11, the obtained Ps amplitudes became small, thus indicating that the aspect ratio of cracks and its variation between high and low tides would also be small. Because land outcrop samples within the fossil seismogenic megasplay fault shows a dominant 

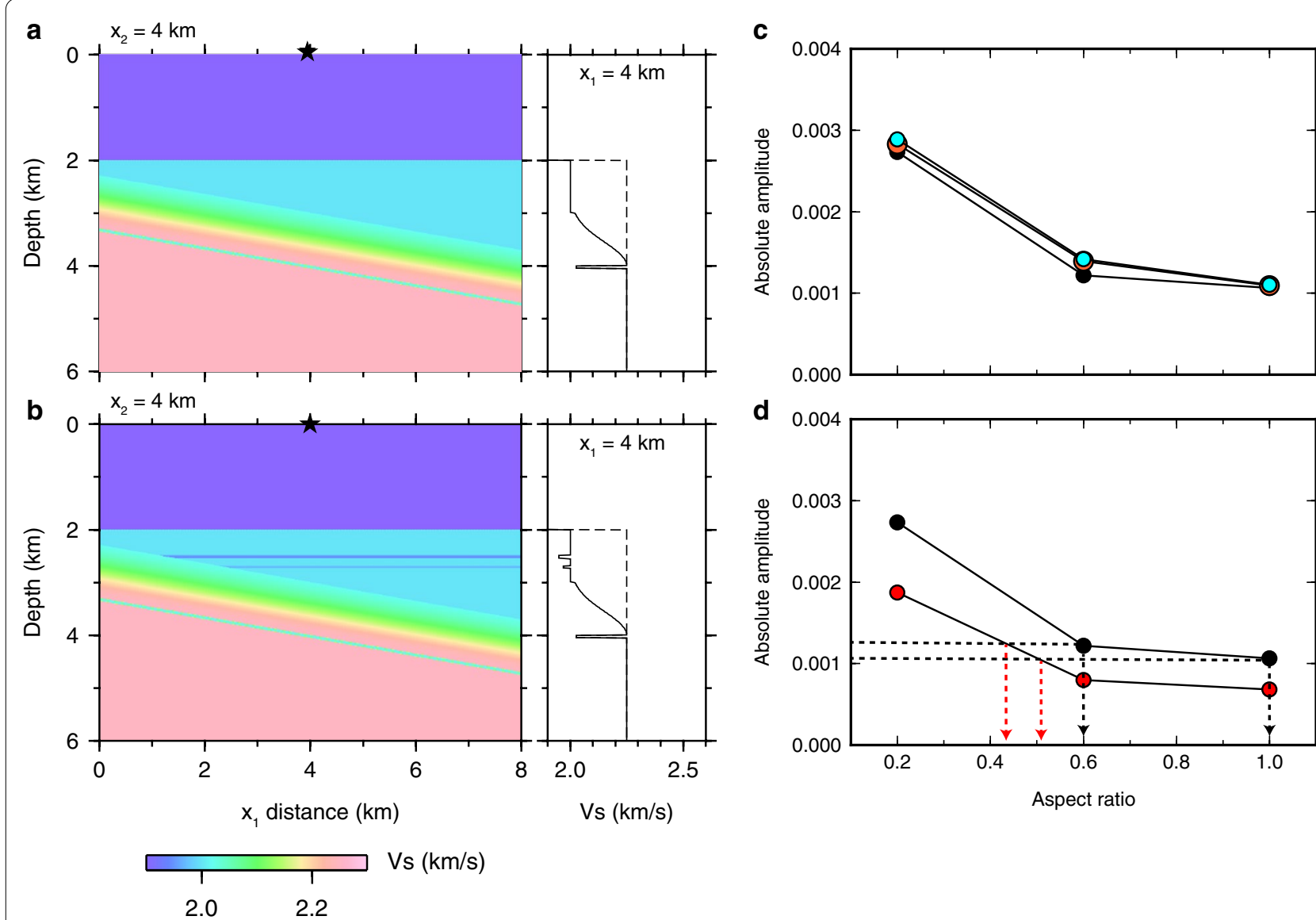

Fig. 11 Numerical simulation results for $P_{s}$ reflected waves in anisotropic media. a Velocity model (1) with a velocity gradient above the megasplay fault at $x_{2}=4 \mathrm{~km}$. Star indicates the source location. Solid and dashed lines in right panel represent 1D velocity model at $\left(x_{1}, x_{2}\right)=(4.0,4.0)$ and the original model, respectively. $\mathbf{b}$ Velocity model (2) with two layers of velocity reductions and a velocity gradient above the megasplay fault. $\mathbf{c} P S$ amplitudes at OBSO2 for (light-blue) model (1), (orange) model (2), and (black) the original model (Fig. 4) at aspect ratio of cracks of 0.2, 0.6, and 1.0. d Ps amplitudes at OBSO2 for (red) porosity of 0.05 and (black) porosity of 0.07 (original model; Fig. 10)

aspect ratio of cracks of 0.1-1.0 (Tsuji et al. 2008), Ps amplitudes obtained in this study can be explained by the $0.1-1.0$ aspect ratio of cracks even if the porosity is slightly smaller than 0.05 .

Moreover, using numerical simulations, we confirm whether the observed $P$ s amplitudes are affected by (1) high velocity anomalies above the megasplay fault, detected by a seismic exploration study (Shiraishi et al. 2019), and (2) small velocity variations at shallow depths (Fig. 11). We examined the following two models: (1) a higher velocity zone above the megasplay fault than that at shallow depths without changing the velocity contrast at the megasplay fault, and (2) two layers above the megasplay fault with velocity reductions of $2.5 \%$ and $1.5 \%$ and thicknesses of $0.05 \mathrm{~km}$ and $0.03 \mathrm{~km}$, respectively. The latter model assumes that other faults are present between the seafloor and the megasplay fault, and their velocities slightly vary due to tidal forces. As a result, the obtained Ps amplitudes were slightly varied in model (1) from those in the original model but the characteristic in which Ps amplitudes increase with decreasing the aspect ratio was not changed, while $P S$ amplitudes were almost unchanged between models (1) and (2) (Fig. 11). This investigation indicates that our results are not significantly affected by the velocity gradient and temporal variations of velocity changes at shallow depth.

\section{Conclusion}

The physical properties of the plate boundary responding to tidal forces are an important factor for understanding the tidal triggering of earthquakes occurring at the plate boundary. Such property changes are attributed to the presence and connection of fluids within the faults, and indicate the existence of mechanically weak interfaces. Through the tidal response of reflection amplitudes, our study identified two such parallel dipping faults around the plate boundary. Slow slip 
events in the shallow Nankai subduction zone have been detected by pore pressure variations at the borehole site (Araki et al. 2017) and also seafloor geodetic observations (Yokota and Ishikawa 2020), and they might have occurred at these multiple weak faults. Additionally, if the physical properties of the weak portions within the megasplay fault have been temporally varied with long-term fluid migration, and if the shear strength within the fault had been very weak, such slow slip events could be possibly triggered by tidal forces.

\begin{abstract}
Abbreviations
OBS: Ocean bottom seismometer; RDW: Radial deconvolved waveform; TDW: Transverse deconvolved waveform; AD-Ps: Amplified differential Ps amplitude; ROV: Remotely operated vehicle; DN: Drilling vessel; R/V: Research vessel; CCF: Cross correlation function; ZZ: Vertical component correlation; $T T$ :Transverse component correlation; RPM: Rotation per minute; RMS: Root mean square; Vp: P-Wave velocity; Vs: S-Wave velocity; CC: Cross correlation coefficient; Ps: $P$-To-s; $R$ shear: Riedel shear.
\end{abstract}

\section{Supplementary Information}

The online version contains supplementary material available at https://doi. org/10.1186/s40623-021-01414-3.

Additional file 1: Figure S1. Parameters for Chikyu on November 20, 2018. a Temporal variation of depth of the drill-bit from the sea surface. The water depth is around 2,000 m. b Temporal variation of RPM (rotation per minute). c Schematic for the geometry of two sensors, drill-bit, and the stationary zones. Figure S2. Transverse-deconvolved waveforms (TDWs) at each OBS. (a-e) Same as Figures $4 \mathrm{~b}$ and CC in $4 d$, but for a OBS01, b OBSO2, c OBSO4, d OBS05, and e OBS06. Figure S3. Location of the center position of Chikyu and the stability of the TDWs. a Dots indicate the center position of Chikyu, which are the same as those in Fig. 2c. Red circle represents the radius of $1 \mathrm{~m}$ from the central location of dots. Regions are divided into eight regions; $A$ : 1 quadrant inside $1 \mathrm{~m}, \mathrm{~B}: 2$ quadrant inside $1 \mathrm{~m}, \mathrm{C}: 3$ quadrant inside $1 \mathrm{~m}, \mathrm{D}: 4$ quadrant inside $1 \mathrm{~m}, \mathrm{E}$ : 1 quadrant outside $1 \mathrm{~m}, \mathrm{~F}: 2$ quadrant outside $1 \mathrm{~m}, \mathrm{G}: 3$ quadrant outside $1 \mathrm{~m}, \mathrm{H}: 4$ quadrant outside $1 \mathrm{~m}$. b Histogram for the Chikyu positions in the four-quadrant. The position was measured at each minute, and the number of 1-min segment during 1-57 Julian days in 2019 was counted. c (top) Waveforms at OBSO3 in which the TDWs are stacked during 1-57 Julian days when the Chikyu is located in the region (black) A, (red) B, (blue) C, and (light-blue) D. (bottom) Same as the top panel, but for the region (black) E, (red) F, (blue) G, and (light-blue) H. Figure S4. Envelope functions at high and low tide. a Same as Fig. 6a, with uncertainties in pink and gray lines for the other OBSs. Arrows indicate large amplitudes at high tide at lag times between 5.5 and 7.5 s. b Same as Fig. 6b, but for OBS06.

\section{Acknowledgements}

We thank crews of D/N Chikyu, R/N Kaimei, and R/V Yokosuka, and Takuya Onodera for supporting OBS deployment. We are grateful for constructive comments from Marine Denolle and one anonymous reviewers, which greatly improved the manuscript. We used the time-series data of drilling parameters during the IODP expedition 358. Bathymetry was acquired by"Research Project for Compound Disaster Mitigation on the Great Earthquakes and Tsunamis around the Nankai Trough Region" of the Ministry of Education, Culture, Sports, Science and Technology (MEXT) of Japan. We used the computer systems of the Earthquake and Volcano Information Center of the Earthquake Research Institute, The University of Tokyo.

\section{Authors' contributions}

T.T. processed the data, and drafted the manuscript. T.K. designed the observation. K.S. prepared the data for reflection profiles and drilling parameters of
Chikyu. S.Y. calculated tidal stress. Y.F. and S.K. contributed to the interpretation. T.T., T.K., K.S., E.A., M.K., Y.S., S.M., Y. N., and S. K. acquired the data underlying this study. All authors read and approved to the final manuscript.

\section{Funding}

This study is supported by MEXT Grant-in-Aid for Scientific Research on Innovative Areas (19H04632)

\section{Availability of data and materials}

Seafloor seismometer data are available from JAMSTEC upon request. The data that support the findings of this study are available from the corresponding author upon reasonable request.

\section{Declarations}

Ethics approval and consent to participate

Not applicable.

\section{Consent for publication}

Not applicable.

\section{Competing interests}

The authors declare no competing interests.

\section{Author details}

1 Japan Agency for Marine-Earth Science and Technology (JAMSTEC), 2-15, Natsushima-cho, Yokosuka 237-0061, Japan. ${ }^{2}$ National Institute of Advanced Industrial Science and Technology, 1-1-1, Umezono, Tsukuba, Ibaraki 305-8560, Japan. ${ }^{3}$ The University of Tokyo, 1-1-1, Yayoi, Bunkyo 113-0032, Japan.

Received: 13 November 2020 Accepted: 1 April 2021

Published online: 15 April 2021

\section{References}

Ammon CJ (1991) The isolation of receiver effects from teleseismic P waveforms. Bull Seismol Soc Am 81:2504-2510

Araki E, Saffer DM, Kopf AJ, Wallace LM, Kimura T, Machida Y, Ide S, Davis E, IODP Expedition 365 shipboard scientists (2017) Recurring and triggered slow-slip events near the trench at the Nankai Trough subduction megathrust. Science 356:1157-1160. https://doi.org/10.1126/science.aan3120

Bartlett WL, Friedman M, Logan JM (1981) Experimental folding and faulting of rocks under confining pressure. Tectonophys 79:255-277

Bensen GD, Ritzwoller MH, Barmin MP, Levshin AL, Lin F, Moschetti MP, Shapiro NM, Yang Y (2007) Processing seismic ambient noise data to obtain reliable broad-band surface wave dispersion measurements. Geophy J Int 169:1239-1260. https://doi.org/10.1111/j.1365-246X.2007.03374.x

Brenguier F, Shapiro NM, Campillo M, Nercessian A, Ferrazzini V (2007) 3-D surface wave tomography of the Piton de la Fournaise volcano using seismic noise correlations. Geophys Res Lett 34:1351-1355. https://doi. org/10.1029/2006GL028586

Brocher TM (2005) Empirical relations between elastic wavespeeds and density in the Earth's crust. Bull Seismol Soc Am 95:2081-2092. https://doi. org/10.1785/0120050077

Clayton R, Engquist B (1977) Absorbing boundary condition for acoustic and elastic wave equations. Bull Seismol Soc Am 67:1529-1540

Cochran ES, Vidale JE, Tanaka S (2004) Earth tides can trigger shallow thrust fault earthquakes. Science 306:1164-1166. https://doi.org/10.1126/scien ce. 1103961

DeMets C, Gordon RG, Argus DF (2010) Geologically current plate motions. Geophys J Int 181:1-80. https://doi.org/10.1111/j.1365-246X.2009.04491.x

Hawthorne JC, Rubin AM (2010) Tidal modulation of slow slip in Cascadia. J Geophys Res 115:B09406. https://doi.org/10.1029/2010JB007502

Houston $\mathrm{H}$ (2015) Low friction and fault weakening revealed by rising sensitivity of tremor to tidal stress. Nat Geosci 8:409-415. https://doi.org/10. 1038/ngeo2419

Ide S (2010) Striations, duration, migration and tidal response in deep tremor. Nature 466:356-360. https://doi.org/10.1038/nature09251 
Ide S, Yabe S, Tanaka Y (2016) Earthquake potential revealed by tidal influence on earthquake size-frequency statistics. Nat Geosci 9:834-837. https:// doi.org/10.1038/ngeo2796

Kimura G, Yamaguchi A, Hojo M, Kitamura Y, Kameda J, Ujiie K, Hamada Y, Hamahashi M, Hina S (2012) Tectonic mélange as fault rock of subduction plate boundary. Tectonophys 568-569:25-38. https://doi.org/10.1016/j. tecto.2011.08.025

Kopf A, Araki E, Toczko S, Expedition 332 Scientists (2011) Proceedings of the IODP. Integrated Ocean Drilling Program

Kopf A, Saffer D, Toczko S, Expedition 365 Scientists (2016) Expedition 365 preliminary report: NanTroSEIZE stage 3: Shallow megasplay Long-Term Borehole Monitoring System (LTBMS) (International Ocean Discovery Program). International Ocean Discovery Program

Kubo H, Nakamura T, Suzuki W, Kimura T, Kunugi T, Takahashi N, Aoi S (2018) Site amplification characteristics at Nankai Seafloor Observation Network, DONET1, Japan, evaluated using spectral inversion. Bull Seismol Soc Am 108:1210-1218. https://doi.org/10.1785/0120170254

Lambert A, Kao H, Rogers G, Courtier N (2009) Correlation of tremor activity with tidal stress in the northern Cascadia subduction zone. J Geophys Res 114:B00A08. https://doi.org/10.1029/2008JB006038

Langston CA (1979) Structure under Mount Rainier, Washington, inferred from teleseismic body waves. J Geophys Res 84:4749-4762

Nagaya M, Oda H, Akazawa H, Ishise M (2008) Receiver functions of seismic waves in layered anisotropic media: application to the estimate of seismic anisotropy. Bull Seismol Soc Am 98:2990-3006. https://doi.org/10.1785/ 0120080130

Nakamura M, Kakazu K (2017) Tidal sensitivity of shallow very low frequency earthquakes in the Ryukyu Trench. J Geophys Res Solid Earth 122:12211238. https://doi.org/10.1002/2016JB013348

Nakanishi A, Kodaira S, Miura S, Ito A, Sato T, Park JO, Kido Y, Kaneda Y (2008) Detailed structural image around splay-fault branching in the Nankai subduction seismogenic zone: results from a high-density ocean bottom seismic survey. J Geophys Res 113:119-214. https://doi.org/10.1029/ 2007JB004974

Nakata R, Suda N, Tsuruoka H (2008) Non-volcanic tremor resulting from the combined effect of Earth tides and slow slip events. Nat Geosci 1:676-678. https://doi.org/10.1038/ngeo288

Nishizawa O (1982) Seismic velocity anisotropy in a medium containing oriented cracks-transversely isotropic case. J Phys Earth 30:331-347

Park JO, Tsuru T, Kodaira S, Cummins PR, Kaneda Y (2002) Splay fault branching along the Nankai subduction zone. Science 297:1157-1160. https://doi. org/10.1126/science.1074111

Rowe CD, Moore JC, Remitti F, the IODP Expedition 343/343T Scientists (2013) The thickness of subduction plate boundary faults from the seafloor into the seismogenic zone. Geology 41:991-994. https://doi.org/10.1130/ G34556.1
Rubinstein JL, Rocca ML, Vidale JE, Creager KC, Wech AG (2008) Tidal modulation of nonvolcanic tremor. Science 319:186-189. https://doi.org/10. 1126/science. 1150558

Saenger EH, Gold N, Shapiro SA (2000) Modeling the propagation of elastic waves using a modified finite-difference grid. Wave Motion 31:77-92

Shapiro NM (2005) High-resolution surface-wave tomography from ambient seismic noise. Science 307:1615-1618. https://doi.org/10.1126/science. 1108339

Shiraishi K, Moore GF, Yamada Y, Kinoshita M, Sanada Y, Kimura G (2019) Seismogenic zone structures revealed by improved 3-D seismic images in the Nankai Trough off Kumano. Geochem Geophys Geosyst 356:1157-1220. https://doi.org/10.1029/2018GC008173

Tanaka S, Sato H, Matsumura S, Ohtake M (2006) Tidal triggering of earthquakes in the subducting Philippine Sea plate beneath the locked zone of the plate interface in the Tokai region, Japan. Tectonophysics 417:69-80. https://doi.org/10.1016/j.tecto.2005.09.013

Tchalenko JS, Ambraseys NN (1970) Structural analysis of the Dasht-e Bayaz (Iran) earthquake fractures. Geol Soc Am Bull 81:41-60

Tonegawa T, Fukao Y, Nishida K, Sugioka H, Ito A (2013) A temporal change of shear wave anisotropy within the marine sedimentary layer associated with the 2011 Tohoku-Oki earthquake. J Geophys Res Solid Earth 118:607-615. https://doi.org/10.1002/jgrb.50074

Tsuji T, Tokuyama H, Costa Pisani P, Moore G (2008) Effective stress and pore pressure in the Nankai accretionary prism off the Muroto Peninsula, southwestern Japan. J Geophys Res 113:320-419. https://doi.org/10. 1029/2007JB005002

Tsuji T, Kamei R, Pratt RG (2014) Pore pressure distribution of a mega-splay fault system in the Nankai Trough subduction zone: Insight into up-dip extent of the seismogenic zone. Earth Planet Sci Lett 396:165-178. https://doi. org/10.1016/j.epsl.2014.04.011

Wang K, Davis EE (1996) Theory for the propagation of tidally induced pore pressure variations in layered subseafloor formations. J Geophys Res 101:11483-11495

Yabe S, Tanaka Y, Houston H, Ide S (2015) Tidal sensitivity of tectonic tremors in Nankai and Cascadia subduction zones. J Geophys Res Solid Earth 120:7587-7605. https://doi.org/10.1002/(ISSN)2169-9356

Yabe S, Tonegawa T, Nakano M (2019) Scaled energy estimation for shallow slow earthquakes. J Geophys Res Solid Earth 124:1507-1519. https://doi. org/10.1029/2018JB016815

Yokota Y, Ishikawa T (2020) Shallow slow slip events along the Nankai Trough detected by GNSS-A. Sci Adv 6:5786

\section{Publisher's Note}

Springer Nature remains neutral with regard to jurisdictional claims in published maps and institutional affiliations.

\section{Submit your manuscript to a SpringerOpen ${ }^{\odot}$ journal and benefit from:}

- Convenient online submission

- Rigorous peer review

- Open access: articles freely available online

- High visibility within the field

- Retaining the copyright to your article

Submit your next manuscript at $\boldsymbol{\nabla}$ springeropen.com 